\title{
The Bacterial Effector AvrB-Induced RIN4 Hyperphosphorylation Is Mediated by a Receptor-Like Cytoplasmic Kinase Complex in Arabidopsis
}

\author{
Ning Xu, ${ }^{1}$ Xuming Luo, ${ }^{1,2}$ Wen Li, ${ }^{1}$ Zongyi Wang, ${ }^{3}$ and Jun Liu ${ }^{1,2}$ \\ ${ }^{1}$ State Key Laboratory of Plant Genomics, Institute of Microbiology, Chinese Academy of Sciences, Beijing, 100101, China; \\ ${ }^{2}$ College of Life Sciences, University of Chinese Academy of Sciences, Beijing, 100049, China; and ${ }^{3}$ Beijing Key Laboratory of \\ Agricultural Product Detection and Control for Spoilage Organisms and Pesticides, Beijing University of Agriculture, Beijing, \\ 102206, China
}

Accepted 27 March 2017.

\begin{abstract}
Bacterial pathogen Pseudomonas syringae delivers diverse type III effectors into host cells to interfere with their immune responses. One of the effectors, AvrB, targets a host guardee protein RIN4 and induces RIN4 phosphorylation in Arabidopsis. Phosphorylated RIN4 activates the immune receptor RPM1 to mount defense. AvrB-induced RIN4 phosphorylation depends on RIPK, a receptor-like cytoplasmic kinase (RLCK). In this study, we found several other RLCKs that were also able to phosphorylate RIN4. We demonstrated that these RLCKs formed a complex with RIPK and were functionally redundant to RIPK. We also found that unphosphorylated RIN4 was epistatic to phosphorylated RIN4 in terms of RPM1 activation. AvrB-induced $R L C K$ gene expression and phosphorylated RIN4-triggered RPM1 activation required RAR1, a central regulator in plant innate immunity. Our results unravel a mechanism in which plants employ multiple kinases to hyperphosphorylate the guardee protein RIN4 to ensure immune activation during pathogen invasion.
\end{abstract}

Plants are constantly exposed to an ever-changing environment that is populated with a plethora of microorganisms. Although plants lack adaptive immune cells, they have evolved effective immune systems to recognize invading microorganisms. The recognition largely relies on two sets of immune receptors, which either tether on plasma membrane or distribute intracellularly (Bonardi et al. 2012; Cui et al. 2015; Macho and Zipfel 2015). The membrane-tethered immune receptors recognize conserved features of microorganisms, such as bacterial flagellin or fungal chitin that are termed pathogen associated molecular patterns (PAMPs), leading to PAMP-triggered immunity (PTI) (Zipfel 2014). The intracellular immune receptor typically contains a coiled-coil or toll/interleukin-1 receptorlike domain, a nucleotide-binding (NB) domain, and a leucinerich repeat (LRR) domain (Bonardi et al. 2012; Chisholm et al. 2006). This set of immune receptors recognizes the effector proteins that are secreted by pathogens, leading to effectortriggered immunity (ETI).

Corresponding author: J. Liu; E-mail: Junliu@im.ac.cn

*The $\boldsymbol{e}$-Xtra logo stands for "electronic extra" and indicates that seven supplementary figures and one supplementary table are published online.

(C) 2017 The American Phytopathological Society
Many phytopathogens harbor hundreds of effectors (Jones and Dangl 2006); whereas, plants carry limited numbers of NBLRR proteins (resistance [R] proteins), which obviously are not enough to recognize all pathogen effectors based on the "genefor-gene" hypothesis (Flor 1971). For example, the model plant Arabidopsis carries only approximately 150 NB-LRRs, but its obligate oomycete pathogen Hyaloperonospora arabidopsidis is predicted to be carrying about equal numbers of effectors (Baxter et al. 2010). The hemibiotrophic bacterial pathogen Pseudomonas syringae pv. tomato DC3000 carries around 30 effectors. Many other oomycete and fungal pathogens carry even more effectors (Baxter et al. 2010). How do plant utilize limited numbers of NB-LRR proteins to recognize various pathogens and their effectors? This enigma is resolved by the "guard hypothesis", which states that plant NB-LRRs recognize pathogens by monitoring (or guarding) effector-caused perturbations of the host targets (the guardee) (Dangl and Jones 2001). One well-characterized guardee is RIN4, a protein of Arabidopsis, which is targeted by at least four bacterial effectors, AvrRpt2, AvrB, AvrRpm1, and HopF2 (Afzal et al. 2013). AvrB is one of the effectors secreted by P. syringae pv. tomato during infection. After AvrB is delivered into the plant cell, RIN4 is rapidly phosphorylated at theronine residue 166 (T166) by the host protein RIPK (Liu et al. 2011). RIN4 T166 phosphorylation triggers activation of RPM1, a NB-LRR protein that specifically recognizes AvrB and AvrRpm1 (Chung et al. 2011; Liu et al. 2011).

Interestingly, the residue S141 of RIN4 is phosphorylated when the plant is treated with flg22 peptide, a peptide derived from bacterial flagella. RIN4 S141 phosphorylation contributes to PTI. However, RIN4pT166 acted epistatically to RIN4pS141 and, essentially, suppressed PTI (Chung et al. 2014). Recently, Lee et al. (2015) reported that RIN4 T21D/S160D/T166D phosphomimetic mutants exhibited enhanced susceptibility, wider stomatal apertures and increased $\mathrm{H}^{+}$-ATPase (AHA1) activity upon surface inoculation with $P$. syringae pv. tomato, indicating that RIN4pT166 negatively affects PTI. These results indicate that RIN4 serves as a "molecular switch" in regulating immune outputs.

AvrB-induced immune response requires the steady-state accumulation of the NB-LRR protein RPM1, which is largely dependent on RAR1 (Holt et al. 2005). RAR1 is a central player in plant immunity. Plants lacking RAR1 not only exhibited reduced basal defense to virulent pathogen $P$. syringae pv. tomato but, also, could not effectively mount a hypersensitive response (HR) to AvrB or AvrRpm1. The latter event is attributed to lower 
levels of RPM1 in rarl plants (Holt et al. 2005). However, in the absence of RPM1, AvrB-caused plant disease susceptibility requires RAR1, demonstrating that RAR1 negatively regulates PTI (Shang et al. 2006). Likewise, in Arabidopsis, constitutive activation of defense responses, such as $\mathrm{H}_{2} \mathrm{O}_{2}$ accumulation and spontaneous cell death, require RAR1, suggesting RAR1 plays other roles in addition to stabilizing $\mathrm{R}$ proteins (Zhou et al. 2008).

RIPK belongs to the receptor-like cytoplasmic kinase (RLCK) subfamily VII in Arabidopsis. Notably, many RLCKs of this family are also heavily involved in immune responses, such as BIK1, PBS1, and PBL13 (Lin et al. 2015; Liu et al. 2013; Zhang et al. 2010). BIK1 plays a positive role in plant immunity. Upon Botrytis cinerea infection, BIK1 transcription is highly upregulated; inactivation of BIK1 causes enhanced susceptibility to necrotrophic fungal pathogens, but the mutant plants are more resistant to $P$. syringae pv. tomato (Veronese et al. 2006). Recent studies show that BIK1 associates with FLS2 or EFR and plays a critical role in PAMP perception (Lin et al. 2013). PBS1 mainly acts in ETI and is specifically required for NB-LRR protein RPS5-mediated resistance. AvrPphB is another $P$. syringae pv. tomato type III effector that is recognized by RPS5 in Arabidopsis. After delivery into host cells, AvrPphB proteolytically cleaves PBS1, resulting in the activation of RPS5 (Shao et al. 2003). Another RLCK protein, PBL13, associates with RBOHD and negatively regulates plant innate immunity before pathogen perception. Knocking out PBL13 leads to elevated expression of PR1, enhanced reactive oxygen species burst, and accelerated flg22-induced activation of mitogen-activated protein kinases, indicating that this kinase plays a negative role in basal defense (Lin et al. 2015).

Previously, we found ripk mutant plants displayed partially compromised RPM1 activation, implying that there might be one or more additional kinases involved in RPM1 activation (Liu et al. 2011). In this study, we identified additional AvrB and RIN4-interacting RLCKs in Arabidopsis. Their expression was also induced by AvrB. Importantly, these kinases could phosphorylate RIN4 at residue 166, presumably contributing to RIN4 hyperphosphorylation. Interestingly, we also found RAR1 was involved in AvrB-induced $R L C K$ gene expression and RIN4 phosphorylation.

\section{RESULTS}

\section{AvrB targets multiple RLCKs in Arabidopsis.}

Previously, we found RIN4 was phosphorylated at T166 by a RLCK, RIPK, but ripk knock-out (KO) lines exhibited partially compromised activation of RPM1 after infection with P. syringae pv. tomato DC3000 (avrB) (Liu et al. 2011). In an attempt to investigate whether there are additional kinases that are capable of phosphorylating RIN4, we performed a yeast two-hybrid $(\mathrm{Y} 2 \mathrm{H})$ screening, using AvrB as a bait, and found that two additional kinases could also interact with AvrB. These two kinases, At5g35580 and At1g69790, belong to RLCK subfamily VII in Arabidopsis, which is comprised of 46 members, including RIPK (Supplementary Fig. S1).

To test whether there are other kinases in this family that are able to interact with AvrB, we cloned all the remaining RLCKs of this subfamily and examined whether they could interact with AvrB in yeast. We identified three more AvrB-interacting RLCKs, namely, At3g01300, At5g15080, and At1g76360, that can strongly interact with AvrB in yeast, but other RLCKs, such as At3g28690, do not interact with AvrB (Fig. 1A). AvrB and RLCK interactions were further confirmed by pull-down assays using recombinant proteins purified from Escherichia coli.(Fig. 1B). To be consistent with the nomenclature employed by Zhang et al. (2010), we named these five RLCKS PBL13
(At5g35580), PBL18 (At1g69790), PBL29 (At3g01300), $P B L 30$ (At5g15080), and PBL31 (At1g76360). Agrobacteriummediated transient expression in Nicotiana benthamiana showed that, similar to RIPK, all five PBS1-like (PBL) kinases were localized to the plasma membrane (Supplementary Fig. S2).

However, of the five AvrB-interacting PBLs, only two interact with RIN4 in Y2H assays (Supplementary Fig. S3). Nevertheless, all five PBLs strongly interacted with RIN4 in $N$. benthamiana by coimmunoprecipitation (Co-IP) and splitluciferase assay (Fig. 1C and D), suggesting that the interactions of RIN4 and the five PBLs may need one or more plant host factors. However, all five PBLs associated with RIPK by Co-IP and split-luciferase assays in $N$. benthamiana (Fig. 2A and B). In addition, we also found, by split-luciferase complementation assay, that the PBLs interacted with each other in $N$. benthamiana, indicating that they may exist as a protein complex in planta.

\section{AvrB-interacting PBLs are involved in RIN4 phosphorylation and RPM1 activation.}

We previously showed that $R I P K$ was transcriptionally induced by $P$. syringae pv. tomato DC3000 (avrB) (Liu et al. 2011). To determine whether AvrB-interacting $P B L$ genes play similar roles as RIPK, we first examined their transcription by quantitative reverse transcription-polymerase chain reaction (qRT-PCR). Like $R I P K$, all $P B L$ transcripts were dramatically upregulated in plants that were inoculated with $P$. syringae pv. tomato DC3000 (avrB), with an exception for PBL30 (Fig. 3A). Notably, $P B L$ transcription levels were also slightly increased upon $P$. syringae pv. tomato DC3000 treatment, suggesting that they may also play roles in basal defense. We next assessed if the five PBLs were able to phosphorylate RIN4 at T166. In vitro kinase assays using recombinant PBLs and RIN4 showed that all these PBLs could phosphorylate RIN4 at T166 (Fig. 3B). This result demonstrates that these PBLs likely are functionally redundant with RIPK in terms of RIN4 phosphorylation.

Because RIN4 phosphorylation at T166 is required for RPM1 activation, we further examined whether these PBLs impact RPM1 activation. We used five T-DNA insertion lines, pbl13 (GABI_586B09) (Lin et al. 2015), pbl18 (Salk_202072), pbl29 (Salk_039402), pbl30 (Salk_067743), and pbl31 (Salk_033107). The RT-PCR validation confirmed that these lines were all KO mutants. Because these genes appear to be functionally redundant, we crossed pbl29 with pbll3 and pbl31 to get double mutants pbl13/pbl29 and pbl29/pbl31. Because there is no T-DNA insertion mutant for ripk in Col-0 background, we designed an artificial microRNA to knock down the expression of RIPK in Col-0 and the pbll3/pbl29 and pbl29/pbl31double mutants (Duan et al. 2008). For each single and triple mutant, we selected two transgenic 35S::amiR-RIPK lines. qRT-PCR results showed that $R I P K$ mRNA levels were significantly reduced in amiR-ripk single mutant lines 3 and 5, pbl triple mutant lines 6 and 7 of pbll3/pbl29/amiR-ripk, and lines 5 and 6 of pbl29/pbl31/amiR-ripk (Supplementary Fig S5). The accumulation of $a m i R-R I P K$ could be detected in transgenic lines by Northern blotting. Notably, we found that among these six $P B L$ gene, RIPK mRNA was the most abundant one in Arabidopsis.

Next, we inoculated wild-type Col- 0 and $p b l$ triple mutant plants with a high titer of $P$. syringae pv. tomato DC3000 (AvrB), at a concentration of $5 \times 10^{7} \mathrm{CFU} / \mathrm{ml}$, and performed an electrolyte leakage analysis, which can reflect the progress of HR-mediated cell death. The result showed that electrolyte leakage was reduced in $p b l$ triple mutants when compared with Col-0, indicating that RPM1 activation-associated HR was attenuated in these mutants (Fig. 4A). Then, we performed growth curve assays to assess their disease resistance. All pbl 
single mutant and amiR-ripk plants exhibited susceptibility to P. syringae pv. tomato DC3000 (avrB) (Fig. 4B), but pbl triple mutants showed more weakly enhanced susceptibility than single or double mutants to $P$. syringae pv. tomato DC3000 (avrB). Notably, pbl13, pbl18, and pbl30 exhibited enhanced resistance to $P$. syringae pv. tomato DC3000 (empty vector [EV]) (Supplementary Fig. S6), indicating that $P B L 18$ and $P B L 30$ may act similarly to $P B L 13$ in basal defense (Lin et al. 2015). pbl single mutants also showed enhanced susceptibility to $P$. syringae pv. tomato DC3000 (avrRpm1); however, we found there were no significant differences between Col- 0 and $p b l$ single mutants when inoculated with $P$. syringae pv. tomato
DC3000 (avrRps4), indicating that PBLs respond to P. syringae pv. tomato DC3000 (AvrB) or DC3000 (AvrRpm1) specifically. These results demonstrated that all five PBLs were specifically involved in RPM1-mediated plant defense responses, leading us to hypothesize a mechanism whereby the plant employs multiple PBLs to interact with and hyperphosphorylate RIN4 to ensure RPM1 activation.

Unphosphorylated RIN4 suppresses RPM1 activation.

Because multiple PBLs were involved in RIN4 phosphorylation, we examined whether the level of phosphorylated RIN4 correlated with RPM1 activation. Previously, we have shown
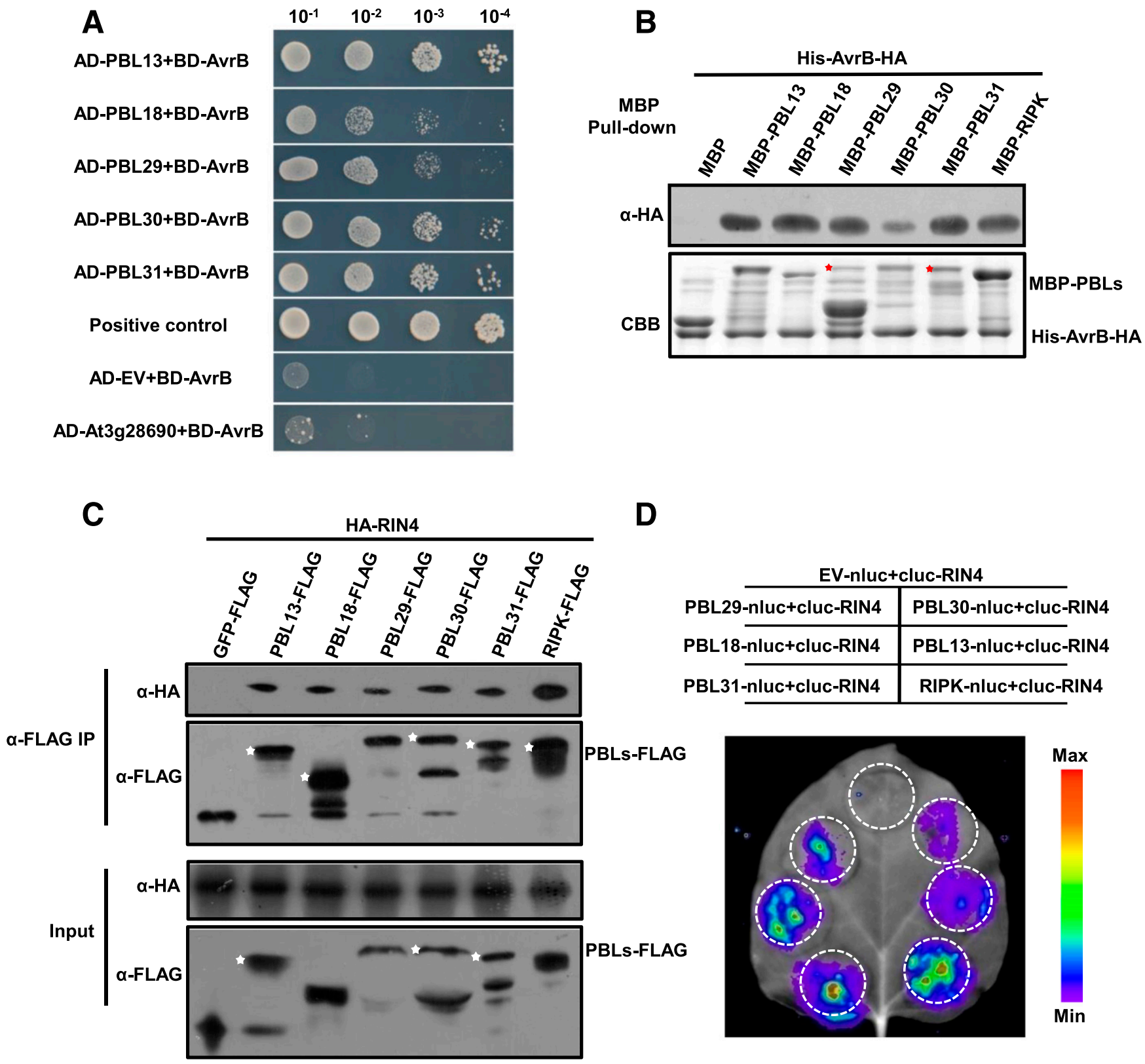

Fig. 1. PBS1-like (PBL) kinases interact with AvrB and RIN4. A, PBLs interacted with AvrB in yeast. $P B L$ genes were cloned in the Matchmaker AD vector and AvrB was cloned in BD vector. pGADT7-T and pGBKT7-53 were used as a positive control. AD-EV+BD-AvrB and AD-At3g28690+BD-AvrB were used as negative controls. B, PBLs interacted with AvrB in vitro. Recombinant maltose-binding protein (MBP)-PBLs and His-AvrB were affinity purified and protein-protein interaction was tested by MBP pull-down assay. CBB, Coomassie brilliant blue staining; a red star indicates the specific MBP-PBL band. C, PBLs interacted with RIN4 in Nicotiana benthamiana by coimmunoprecipitation assay (Co-IP). N. benthamiana leaves were coinfiltrated with agrobacterium carrying $35 S:: P B L-3 \times F L A G$ and $35 S:: 3 \times H A-R I N 4$. Infiltrated leaves were harvested two days later and a Co-IP assay was performed, using ANTI-FLAG M2 affinity gel. A white star indicates the specific PBL-FLAG band. D, PBLs interacted with RIN4 in $N$. benthamiana by a split-luciferase complementation assay. $N$. benthamiana leaves were coinfiltrated with agrobacterium carrying $35 S:: P B L-n l u c$ and $35 S:: c l u c-R I N 4$. A luciferase complementation imaging assay was performed two days later. nluc, N-terminal fragment of firefly luciferase; cluc, C-terminal fragment of firefly luciferase; EV, empty vector. 
npro::pRIN4 (RIN4pT166) transgenic plants were dwarf in rin4/rps 2 backgrounds, due to the constitutive activation of RPM1 (Liu et al. 2011). In order to test if unphosphorylated RIN4 affects RPM1 activation, we crossed npro::pRIN4 (rin4/rps2) plant with rps 2 plants. Interestingly, we found that $r p s 2 / r p s 2 R I N 4 / R I N 4$ $p R I N 4 / p R I N 4$ plants displayed the wild-type phenotype, implying that endogenous unphosphorylated RIN4 may suppress RIN4pT166 in the activation of RPM1 (Fig. 5A). To determine how unphosphorylated RIN4 suppresses RPM1 activation, we transiently expressed different ratios of RIN4 versus RIN4pT166 in $N$. benthamiana. The result showed that RIN4 efficiently suppressed RIN4pT166-induced RPM1 activation at ratios above 1:3 (Fig. 5B).

\section{AvrB-induced $P B L$ expression is dramatically} reduced in rarl mutant plants.

It was worth noting that expression of all the AvrB-interacting PBLs was induced by $P$. syringae pv. tomato DC3000 (AvrB) treatment (Fig. 3A). It has been reported that AvrB also associates with RAR1, a key immune regulator (Shang et al. 2006). de Torres et al. (2003) showed that RIPK induction was reduced in a rarl-28 mutant after $P$. syringae pv. tomato DC3000 (avrRpm1) treatment, which inspired us to test if expression of the other AvrB-interacting PBLs was also reduced in rarl plants. First, we examined the expression of $R A R l$ by various treatments. qRT-PCR results showed that $R A R 1$ expression was induced by $P$. syringae pv. tomato DC3000, DC3000 (avrB), and DC3000 (hrcC), and flg22 treatments (Fig. 6A). Then, we examined $P B L$ expression in rarl mutant. As shown in Figure 6B, the transcription levels of five $P B L s$ were remarkably reduced in rarl after inoculation with $P$. syringae pv. tomato DC3000 (avrB). In addition, we also observed that the expression of PBL18, PBL29, and PBL31 was slightly decreased in rpm1 mutants (Supplementary Fig S7), implying that RAR1 and probably RPM1 play critical roles in AvrB-induced $P B L$ expression.

To validate the qRT-PCR results at the protein level, we examined the phosphorylation of RIN4T166 in rarl, rpml, and rarl/rpml double mutants after pathogen treatments. Immunoblot assays showed that RIN4T166 phosphorylation was reduced significantly in rarl and rarl/rpml after $6 \mathrm{~h}$ of $P$. syringae pv. tomato DC3000 (avrB) treatment when compared with Col-0. RIN4 phosphorylation was also slightly reduced in rpml (Fig. 7A and B). These data demonstrate that AvrB-induced PBL expression and RIN4 phosphorylation are affected by RAR1 and RPM1. In addition, we also detected the RIN4 phosphorylation levels in $p b l$ triple mutants. The results showed that RIN4 phosphorylation was also decreased in the triple mutants pbl13/pbl29/amiR-ripk and pbl29/pbl31/amiR-ripk (Fig. 7A and B), implying that other PBLs are indeed involved in RIN4 phosphorylation in addition to RIPK.

\section{DISCUSSION}

RIN4 is a unique immune protein that, so far, it has been shown to be targeted by at least four bacterial type III effectors, AvrRpt2, AvrB, AvrRpm1, and HopF2 (Afzal et al. 2013; Khan et al. 2016). Recent studies indicate that RIN4 is a bona fide virulence target of the above effectors. The modifications of RIN4 through cleavage and phosphorylation eventually lead to immune responses (Afzal et al. 2013; Chung et al. 2011; Coaker et al. 2005; Liu et al. 2011). However, RIN4 is a negative regulator in PTI, as overexpressing RIN4 in Arabidopsis reduced resistance to $P$. syringae pv. tomato DC3000 hrcC, a mutant of DC3000 that is deficient in the type III secretion system, whereas RIN4 KO plants display enhanced resistance to this pathogen (Kim et al. 2005; Liu et al. 2009). RIN4mediated suppression of the PTI response depends on either its

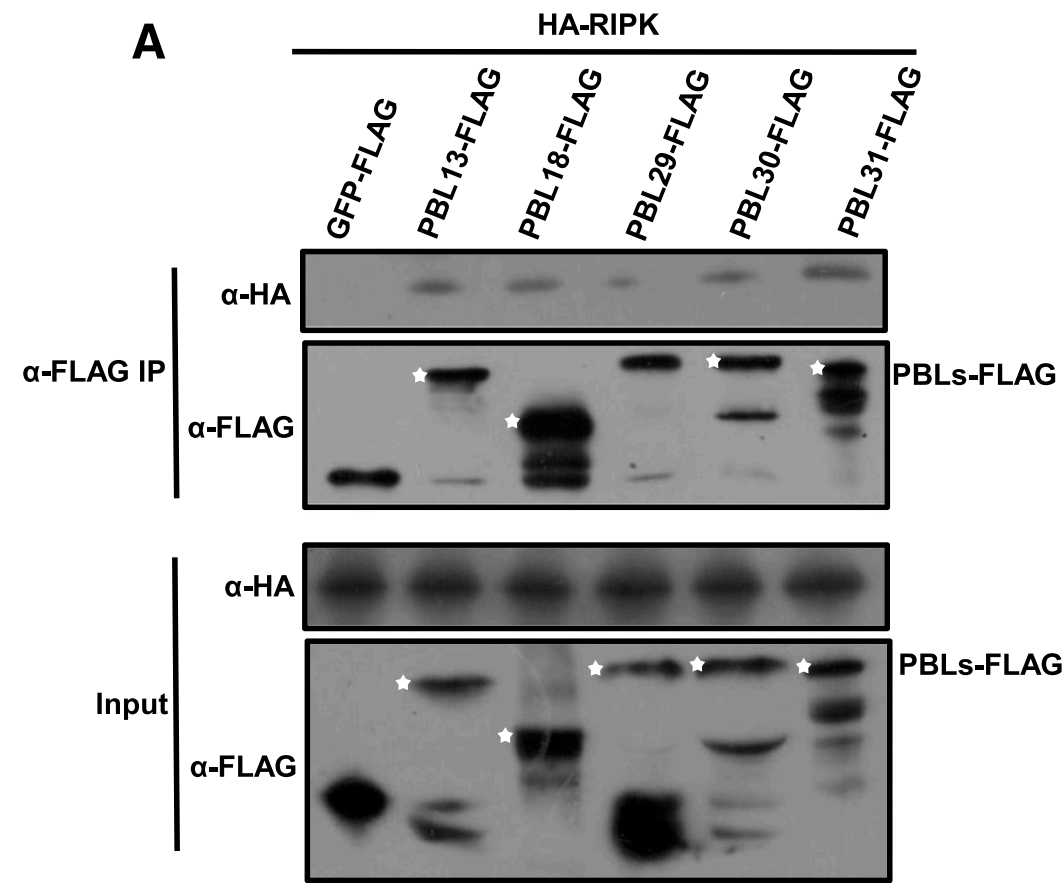

B

\begin{tabular}{r|l}
\hline $\begin{array}{r}\text { EV-nluc+cluc-RIPK } \\
\hline \text { PBL30-nluc+cluc-RIPK }\end{array}$ & PBL29-nluc+cluc-RIPK \\
\hline PBL13-nluc+cluc-RIPK & PBL31-nluc+cluc-RIPK \\
\hline
\end{tabular}

Fig. 2. PBS1-like (PBL) kinases interact with RIPK, a receptor-like cytoplasmic kinase. A, PBLs interacted with RIPK in Nicotiana benthamiana by coimmunoprecipitation (Co-IP). $N$. benthamiana leaves were coinfiltrated with agrobacterium carrying $35 S:: P B L-3 \times F L A G$ and $35 S:: 3 \times H A-R I P K$. Infiltrated leaves were harvested 2 days later, and a Co-IP assay was performed using ANTI-FLAG M2 affinity gel. A white star indicates the specific PBL-FLAG band. B, PBLs interacted with RIPK in $N$. benthamiana by split-luciferase complementation. $N$. benthamiana leaves were coinfiltrated with agrobacterium carrying $35 S:: P B L-n l u c$ and $35 S:: c l u c-R I P K$. A luciferase complementation imaging assay was performed two days later. 
evolutionarily conserved NOI domain, its proper subcellular localization. or both (Afzal et al. 2011).

The modification of specific residues of RIN4 could also contribute to either PTI or ETI. For example, phosphorylation of RIN4 T166 is essential for RPM1 activation (Liu et al. 2011).
RIN4 could be ADP-ribosylated by HopF2, and such modification may contribute to HopF2-promoted and RIN4-dependent $P$. syringae pv. tomato growth (Wang et al. 2010). Recently, Chung and colleagues (2014) reported that RIN4pT166 contributed to the suppression of PTI in Arabidopsis, but the residue
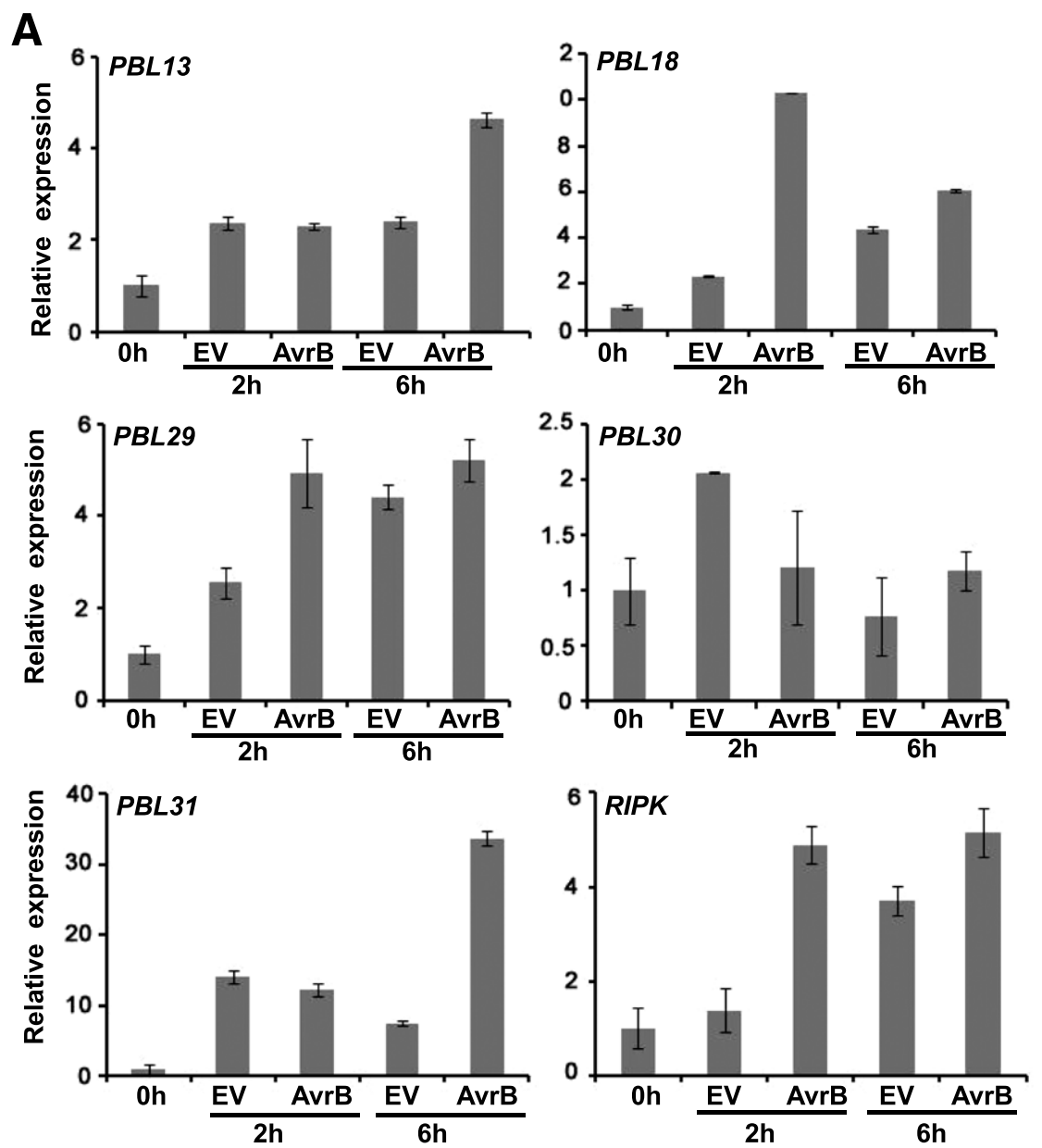

B

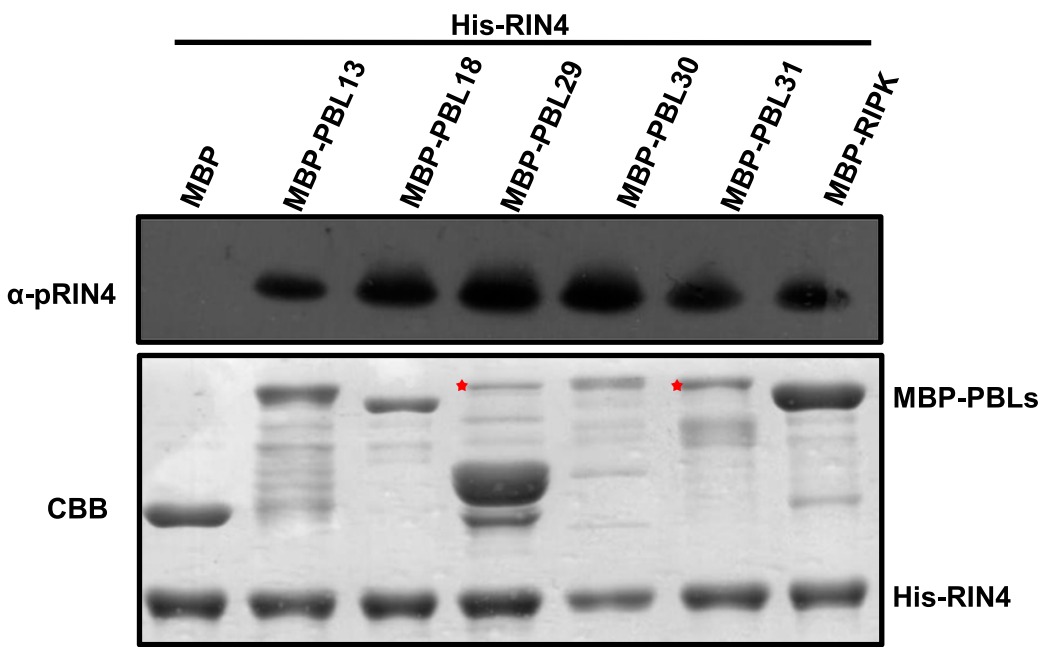

Fig. 3. PBS1-like (PBL) kinases can phosphorylate RIN4 at theronine residue 166 (T166). A, $P B L$ gene expression was induced by AvrB. Col-0 leaves were syringe infiltrated with Pseudomonas syringae pv. tomato DC3000 and DC3000 (avrB) at a concentration of $5 \times 10^{7} \mathrm{CFU} / \mathrm{ml}$ and were sampled at 0,2 , and $6 \mathrm{~h}$ postinoculation for quantitative reverse transcription-polymerase chain reaction (qRT-PCR) analysis. Error bars represent means \pm standard deviation for qRTPCR $(n=3)$. B, PBLs can phosphorylate RIN4 T166 in vitro. Recombinant maltose-binding protein (MBP)-PBLs $(1 \mu \mathrm{g})$ were incubated with $3 \mu \mathrm{g}$ of recombinant His-RIN4 in the kinase activity assays. The bands in the upper panel were blotted with anti-pRIN4T166 antibody ( $\alpha$-pRIN4). CBB, Coomassie brilliant blue staining. A star indicates the specific MBP-PBL band. 
S141 phosphorylation enhanced PTI response, although the kinase that was responsible for RIN4 phosphorylation at S141 is unknown (Chung et al. 2014). Notably, functions of RIN4pS141 were repressed by RIN4pT166 during PTI. Likewise, Lee et al. (2015) also found RIN4 T21D/S160D/T166D enhanced plant susceptibility to $P$. syringae pv. tomato, further underpinning the negative role of RIN4pT166 in PTI.

Previously, we showed that RIN4 phosphorylation at T166 required RIPK. However, ripk mutant plants displayed only partially compromised RPM1 activation. Therefore, the additional PBLs identified in this study partially resolved this observation. Indeed, our $p b l$ triple mutant plants displayed compromised RPM1 activation (Fig. 4). However, an interesting question is why do plants deploy multiple kinases to phosphorylate RIN4? The plausible explanation probably is that plants must ensure RIN4 to be hyperphosphorylated in order to trigger RPM1 activation once the plant perceives high levels of AvrB or AvrRpm1. Because HR is a strong immune response, usually being at the cost of plant growth, plants may activate NB-LRRs only when there are high titers of pathogens and the cognate effectors. Therefore, the high levels of phosphorylated RIN4 may essentially require multiple kinases.

By performing bioinformatics analyses on RIN4 homologs from different plant species and the NOI proteins from Arabidopsis, Sun et al. (2014) suggested that RIN4 is an intrinsically disordered protein with binding promiscuity and plasticity to interacting partners. This structure enables RIN4 to interact with multiple immune proteins and to be positioned at pivotal sites to act as hubs in various pathways. As such, RIN4 interacts with multiple host proteins even when there is no pathogen infection. Probably because of the same reason, RIN4 is targeted by many pathogen-secreted effectors. Therefore, the state

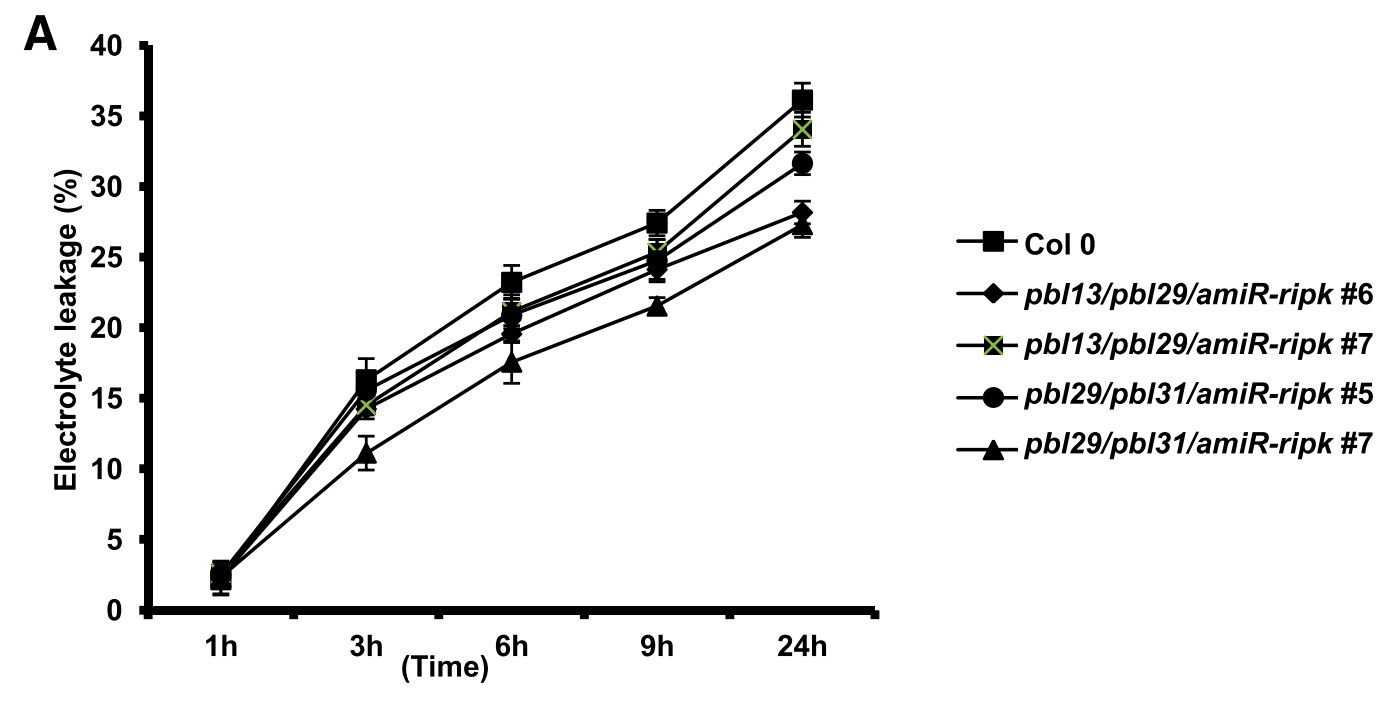

B

Pst DC3000 (avrB)

C

Pst DC3000 (avrB)
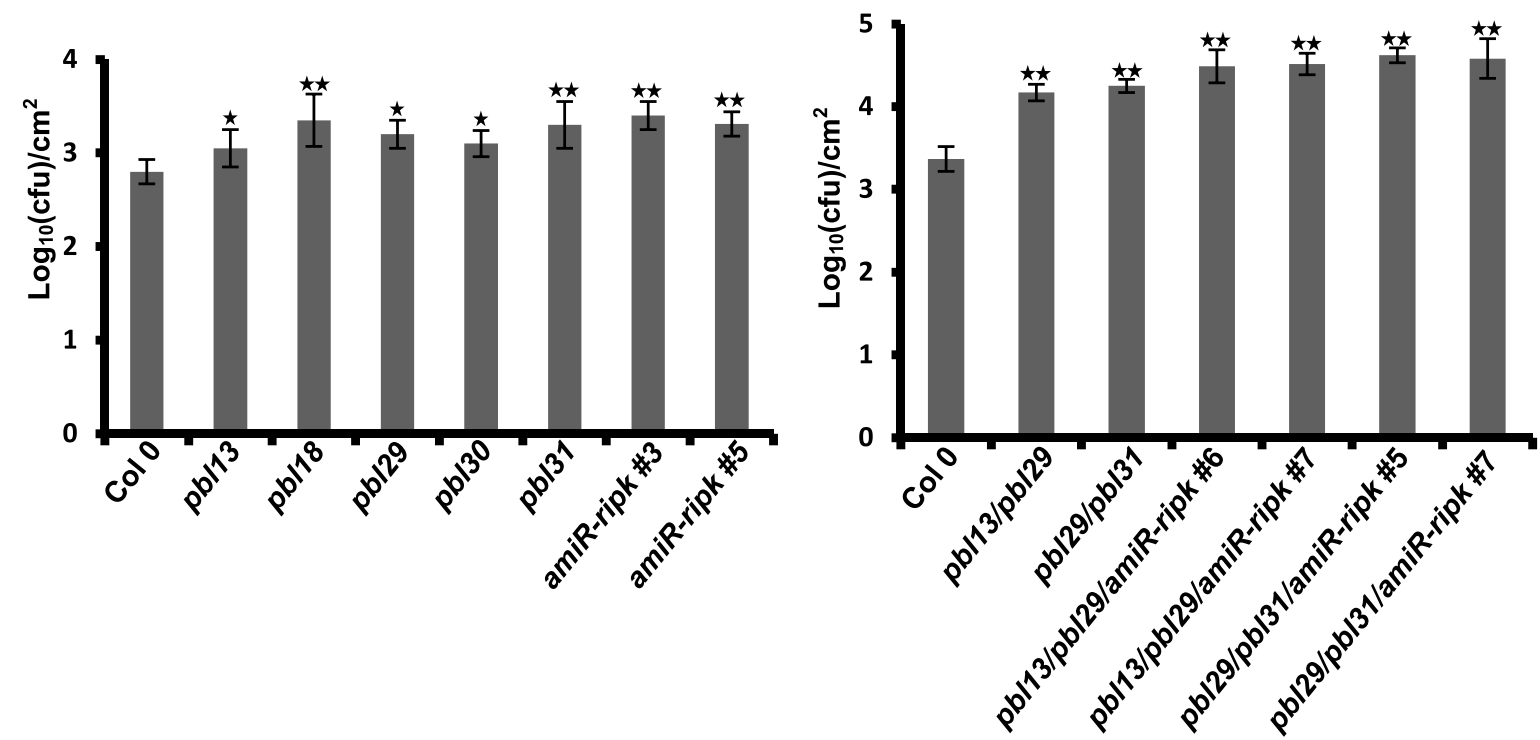

Fig. 4. The $p b l$ triple mutant plants displayed compromised RPM1-mediated disease resistance. A, The $p b l$ triple mutants showed reduced electrolyte leakage as compared with Col-0 after Pseudomonas syringae pv. tomato DC3000 (avrB) treatment. Four-week-old plants were infiltrated with $5 \times 10^{7}$ CFU of P. syringae pv. tomato DC3000 (avrB) per milliliter. Electrolyte leakage was monitored in three replicates at the indicated time points. Data are means \pm standard deviation (SD) $(n=3)$. B and $\mathbf{C}$, The $p b l$ single, double, and triple mutants exhibited enhanced susceptibility to $P$. syringae pv. tomato DC3000 (avrB). Four-week-old plants were infiltrated with $5 \times 10^{4} \mathrm{CFU}$ of $P$. syringae pv. tomato DC3000 (avrB) per milliliter. Bacterial population sizes were quantified 3 days postinoculation. The data shown are representative of three independent experiments with similar results. Asterisks indicate significant differences from wild type at $P<0.01(* *)$ and $P<0.05(*)$. Data are means \pm SD $(n=6)$. 
of RIN4 is crucial in immune response. In fact, recent discoveries demonstrated that either RIN4pS141 or RIN4 ${ }^{\mathrm{P} 149 \mathrm{~V}}$ could counteract the roles of RIN4pT166, possibly by changing the molecular conformation, indicating that the intermolecular switch controls defense outputs (Chung et al. 2014; Li et al. 2014; Lee et al. 2015). In other words, the modification of RIN4 has to be tightly controlled to avoid improper activation by other signaling pathways.

Interestingly, our in vivo experiments revealed that unphosphorylated RIN4 effectively suppressed RIN4pT166-mediated RPM1activation in N. benthamiana (Fig. 5). Furthermore, the RIN4pT166 transgenic plant was phenotypically normal as wild-type plants when the plants genetically carried RIN4, indicating that RIN4 is epistatic to phosphorylated RIN4 in terms of RPM1 activation. These results also suggest that RIN4pT166 must accumulate at a high level to override unphosphorylated RIN4 to activate RPM1. Because RIN4 is known to be playing a negative role in PTI, its suppression on RIN4pT166 suggests the intermolecular balance of RIN4 could also control the defense outputs.

Further, we found that phosphorylated RIN4-caused immune response was fine-tuned at the transcription level by controlling expression of PBLs. After perceiving AvrB, AvrB-interacting $P B L s$ expression was significantly upregulated (Fig. 3A). Interestingly, plant key immune regulator RAR1 and likely RPM1 were involved in $P B L$ expression (Fig. 6B). It is known that RAR1 was required for AvrB-orchestrated suppression of callose deposition induced by flg22, acting as a negative regulator in AvrB-suppressed PTI (Shang et al. 2006). de Torres et al. (2003) showed that RIPK induction was reduced in a rarl

A

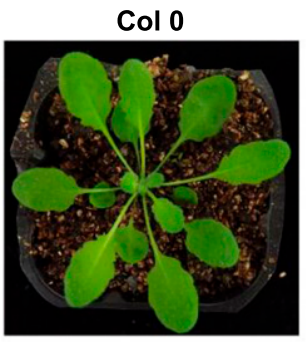

npro::pRIN4(r2/r4)
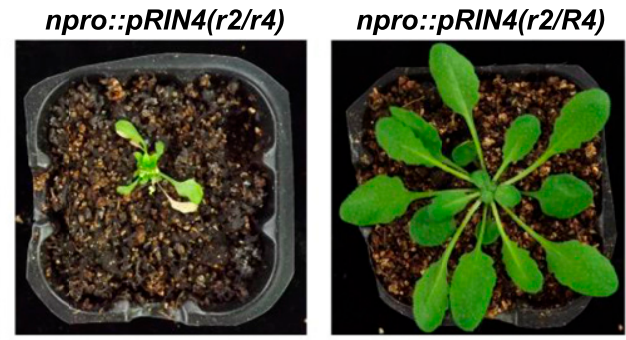

B
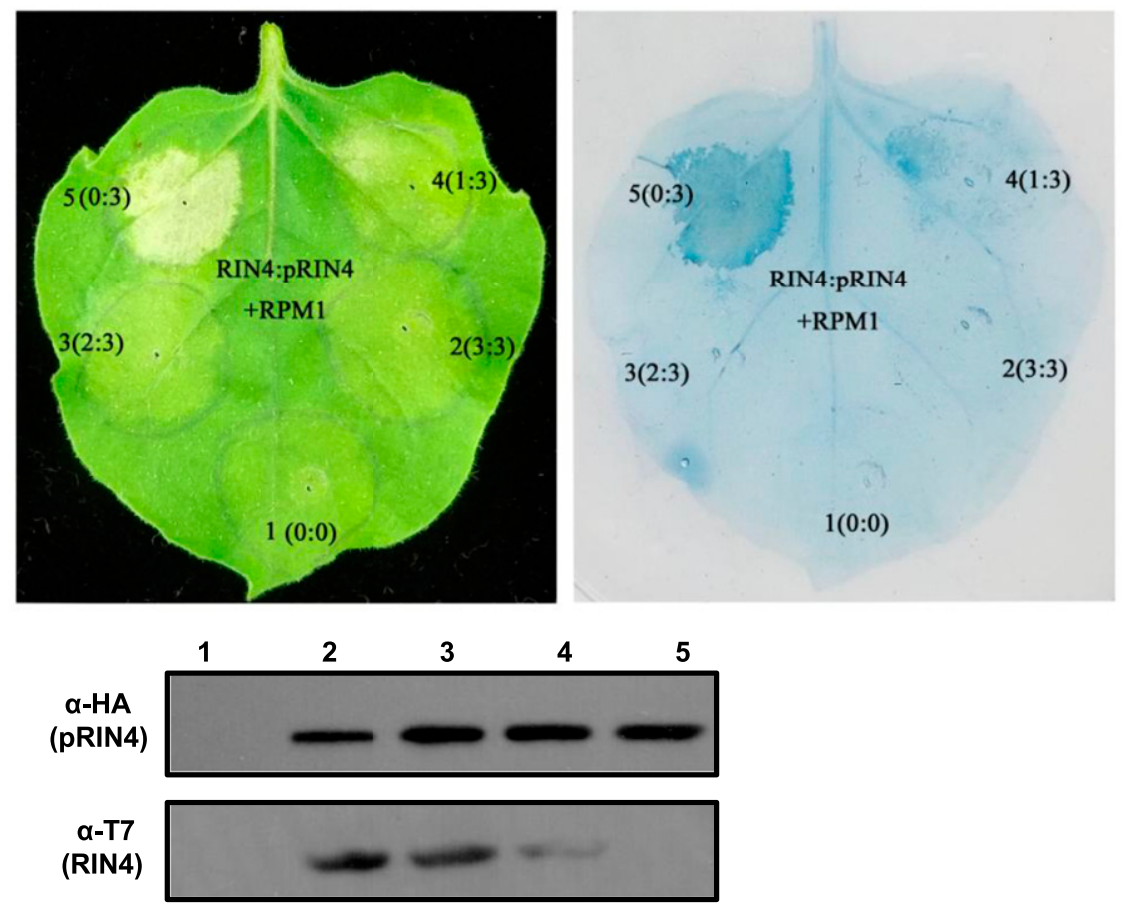

a-MYC

(RPM1)

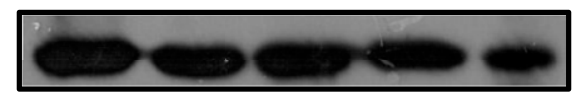

Rubisco

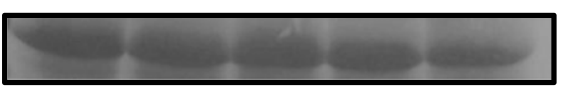

Fig. 5. RIN4 is epistatic to RIN4pT166. A, Wild-type (endogenous unphosphorylated) RIN4 suppressed dwarf phenotype of $n p r o:: p R I N 4(r 2 / r 4)$ plants. $n p r o::$ pRIN4(r2/r4) transgenic plants were crossed with rps2. The genotype of F2 generation plants was identified. npro::pRIN4(r2/R4) plants showed a wild-type phenotype. Photographs were taken at 5 weeks after germination. B, Unphosphorylated RIN4 repressed pRIN4-triggered RPM1 activation in Nicotiana benthamiana. Agrobacterium carrying 35S::T7-RIN4 and 35S::HA-pRIN4 was cotransformed in N. benthamiana leaves at ratios of $0: 3$, 1:3, 2:3, and 3:3 (RIN4/pRIN4). The bacteria titer was adjusted to equal amounts with agrobacterium carrying empty vector or $35 S:: T 7-R I N 4$. $35 S:: H A-p R I N 4$ and $35 S:: R P M 1$ $M Y C$ was infiltrated at a concentration of $1.5 \times 10^{8}$ and $2 \times 10^{8} \mathrm{CFU} / \mathrm{ml}$, respectively. Leaves were sampled for immunoblot analysis at $48 \mathrm{~h}$ postinoculation (hpi) and were stained with trypan blue at $60 \mathrm{hpi}$. 
mutant after $P$. syringae pv. tomato DC3000 (avrRpm1) treatment. These results suggest that RAR1 is involved in RPM1mediated immune activation. Notably, AvrB associates with RAR1 protein complex, which may potentially interfere with RAR1-mediated signaling pathways. Our results showed that $P B L$ expression was reduced in rarl mutant plants after treatment with AvrB. As a result, phosphorylation of RIN4 was reduced and RPM1 activation was attenuated.
In plants that lack RPM1, RAR1 is a negative regulator of PTI and plays a critical role in AvrB-suppressed callose deposition (Shang et al. 2006). Importantly, AvrB-suppressed callose deposition required RIN4pT166 (Chung et al. 2014). These results imply that PBL-mediated RIN4 phosphorylation at T166 may link AvrB virulence function to RAR1. We hypothesize a scenario in which AvrB induces expression of PBLs through RAR1 and, subsequently, PBLs phosphorylate RIN4, resulting
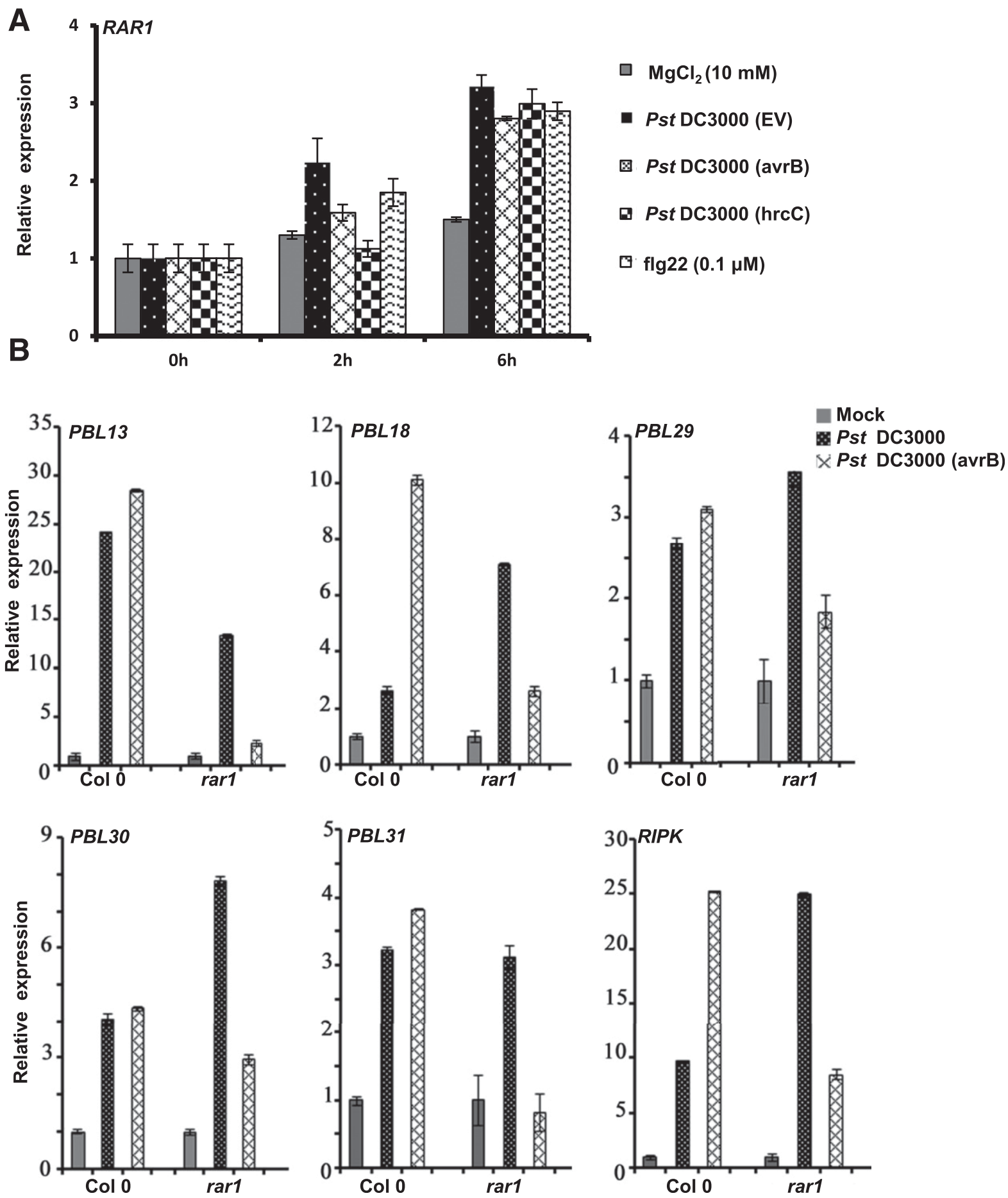

Fig. 6. AvrB-induced PBS1-like kinase gene expression is reduced in rarl. A, RAR1 expression was induced by pathogen treatments. Col-0 leaves were syringe-infiltrated with $10 \mathrm{mM} \mathrm{MgCl}_{2}$, Pseudomonas syringae pv. tomato DC3000, DC3000 (avrB), and DC3000 (hrcC) at concentrations of $5 \times 10^{7} \mathrm{CFU} / \mathrm{ml}$ or $0.1 \mu \mathrm{M}$ flg22. Samples were harvested at 0,2 , and $6 \mathrm{~h}$ postinoculation (hpi) for quantitative reverse transcription-polymerase chain reaction (qRT-PCR) analysis. Error bars represent means \pm standard deviation for qRT-PCR $(n=3)$. B, $P B L$ genes expression was significantly reduced in rarl after $P$. syringae pv. tomato DC3000 (avrB) treatment. Plants were infiltrated with $5 \times 10^{7}$ CFU of $P$. syringae pv. tomato DC3000 (EV) and DC3000 (avrB) per milliliter and were sampled at 0 and 4 hpi. Error bars represent means \pm standard deviation for qRT-PCR $(n=3)$. 
in the repression of PTI. These results can help to explain how RAR1 plays a negative role in PTI. Although we observed AvrBinduced $P B L$ expression was affected by RAR1, the underlying mechanism remained unclear. As AvrB could target RAR1 complexes, the interaction may contribute to RAR1-mediated $P B L$ expression. However, in the presence of RPM1, AvrBinduced RIN4 hyperphosphorylation eventually caused RPM1 activation.

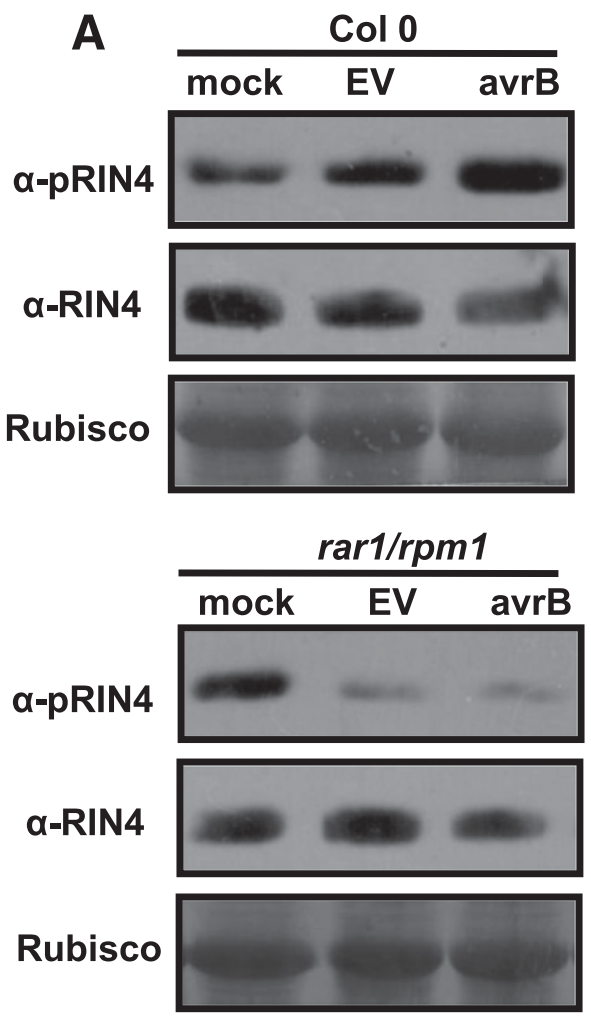

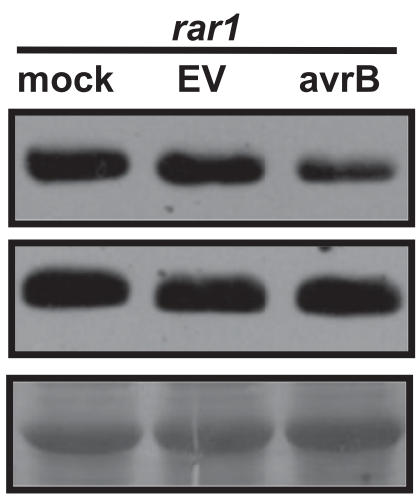
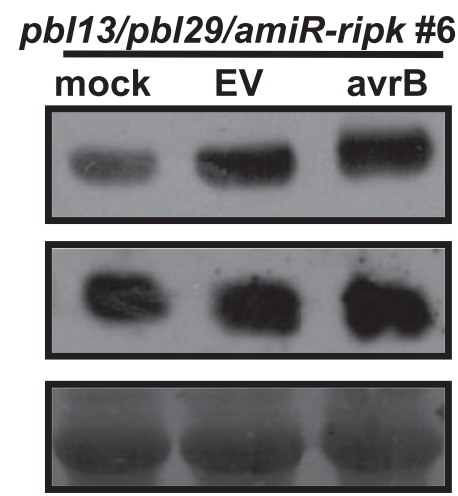
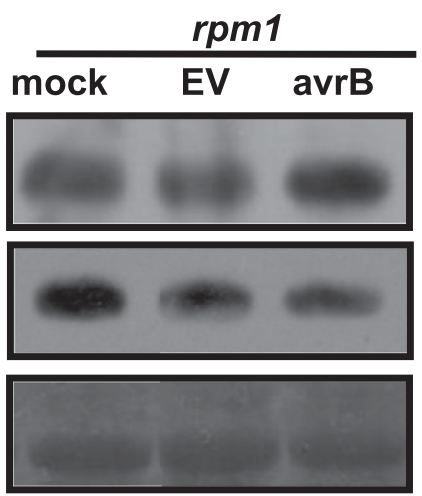

\section{pb/29/pb/31/amiR-ripk \#5} mock EV avrB
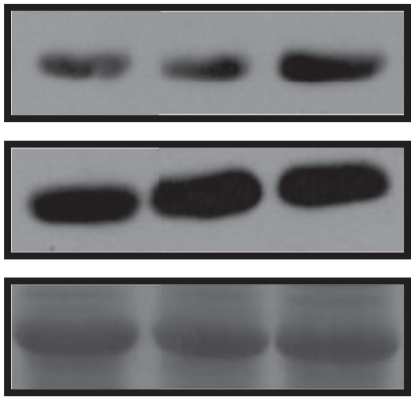

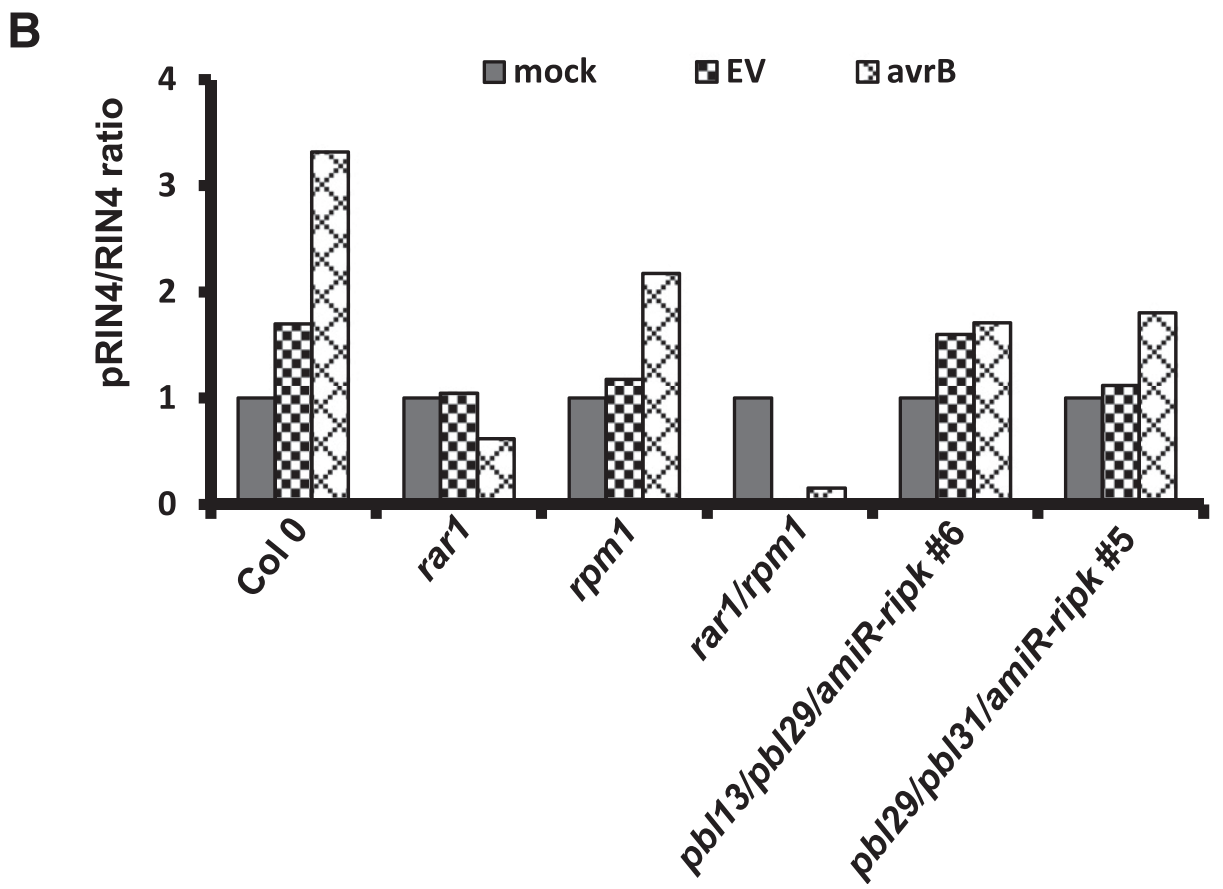

Fig. 7. RIN4 phosphorylation is reduced in $\operatorname{rarl} 1, \mathrm{rpml}, \mathrm{rarl} / \mathrm{rpm} 1$, and $\mathrm{pbl}$ triple mutants. A, RIN4 phosphorylation levels were detected in $\mathrm{rarl}$, rarl/rpml, and $p b l$ triple mutants by immunoblotting with $\alpha$-pRIN4 T166 antibody and $\alpha$-RIN4 antibody. Plants were infiltrated with $5 \times 10^{7} \mathrm{CFU}$ Pseudomonas syringae pv. tomato DC3000 (EV) and DC3000 (avrB) per milliter and were sampled at $6 \mathrm{~h}$ postinoculation. Mock was infiltrated with $10 \mathrm{mM} \mathrm{MgCl}_{2}$. B, Quantification of immunoblotting signals shown in A by Image $\mathbf{J}$ software. The band intensity for phosphorylated RIN4 and RIN4 were scanned and calculated. The ratio stands for pRIN4/RIN4. The data shown are representative of three independent experiments with similar results. 
It is known that RAR1 stabilizes NB-LRR proteins and affects their activities. Therefore, it was expected that RIN4pT166 could not activate or delayed activation of RPM1 in rarl mutant plants. However, RARl expression could be induced by various pathogen treatments and the AvrB-interacting $P B L$ expression was reduced in rarl (Fig. 6). Thus, RAR1 likely serves as a molecular hub in controlling the outputs of defense strength in both PTI and ETI and plays dual roles in RIN4 phosphorylation and RPM1 stabilization in Arabidopsis.

Taken together, our results demonstrated that AvrB and RIN4 associated with multiple PBLs in Arabidopsis, which eventually resulted in hyperphosphorylation of RIN4 after perceiving a high titer of AvrB or AvrRpm1. We propose a scenario that AvrB targets RAR1 to suppress PTI by inducing $P B L$ expression and RIN4 phosphorylation at T166 in the plants that lack RPM1; however, in the plants that genetically carry RPM1, RIN4 hyperphosphorylation (pT166), by contrast, activates RPM1, leading to ETI in which RPM1 activation is decided by the ligand states (RIN4 versus RIN4pT166 in this case). This phenomenon may also apply to other immune receptor activations.

\section{MATERIALS AND METHODS}

\section{Plant materials and growth conditions.}

Arabidopsis thaliana T-DNA mutants used in this study including pbl29 (Salk_039402), pbl30 (Salk_067743), pbl18 (Salk_202072), pbl13 (GABI_586B09) and pbl31 (Salk_033107) were obtained from the Arabidopsis Biological Resource Center. Homozygous seeds for rpml and rarl-28 were previously described (Grant et al. 1995; Tornero et al. 2002). npro::pRIN4 (RIN4pT166) transgenic plants were generated in previous study (Liu et al. 2011). All the mutants mentioned above were in the Col-0 background and were confirmed by RT-PCR for homozygous KOs. All plants were grown at $23^{\circ} \mathrm{C}$ under a 10 -h-light and 14-h-dark cycle and 4- to 5-week-old plants were used for experiments.

\section{Y2H.}

The $\mathrm{Y} 2 \mathrm{H}$ experiments were performed following the manufacture's protocol (Matchmaker, Clontech Laboratories, Inc.). The coding sequences of PBL29, PBL30, PBL18, PBL13, and $P B L 31$ were cloned into pGADT7 vector; $A v r B$ and $R I N 4$ were cloned into pGBKT7 vector. pGADT7-T and pGBKT7-53 were used as positive controls. Cotransformations of pGADT7-EV with pGBKT7-RIN4 or pGBKT7-AvrB were used as negative controls. The yeast strain AH109 was transformed with corresponding constructs and was plated onto synthetic dextrose agar lacking leucine, tryptophan, and histidine. The yeast growth was examined as previously described (Liu et al. 2011)

\section{Recombinant protein pull-down assays.}

Recombinant proteins of AvrB and PBLs were prepared as previously described (Liu et al. 2011). For pull-down assays, His-AvrB-HA (hemagglutinin), maltose-binding protein (MBP), MBP-PBL29, MBP-PBL30, MBP-PBL18, MBP-PBL31, and MBP-RIPK (3 $\mu \mathrm{g}$ each) were incubated with Amylose resin $(10 \mu \mathrm{l})$ in TEN buffer $(20 \mathrm{mM}$ Tris-HCl, $\mathrm{pH} 7.4,100 \mathrm{mM} \mathrm{NaCl}$, and $0.2 \%$ Triton $\mathrm{X}-100$ ) on an earthquake shaker for $1 \mathrm{~h}$, followed by two in-tube washes. The mixture was transferred to a micro spin column (Bio-Rad) and was washed twice with $500 \mu \mathrm{l}$ of NETN300 (20 mM Tris-HCl, pH 7.4, $300 \mathrm{mM} \mathrm{NaCl}$, and $0.5 \%$ NP-40). The proteins were eluted from the beads with $10 \mathrm{mM}$ maltose. The presence of His-AvrB-HA was detected by anti-HA.

\section{Co-IP assay.}

The indicated constructs were transiently expressed in $N$. benthamiana. Two days later, total protein was extracted for
Co-IP with the extraction buffer $(50 \mathrm{mM}$ Tris- $\mathrm{HCl}, \mathrm{pH} 7.5$, $150 \mathrm{mM} \mathrm{NaCl}, 0.1 \%$ Triton, $0.2 \% \mathrm{NP}-40,6 \mathrm{mM}$ 2-mercaptoethanol, proteinase inhibitor cocktail). Total protein was incubated with $20 \mu \mathrm{l}$ of ANTI-FLAG M2 affinity gel (Sigma) for $1 \mathrm{~h}$ and were washed five times with washing buffer $(50 \mathrm{mM}$ Tris-HCl, $\mathrm{pH} 7.5,150 \mathrm{mM} \mathrm{NaCl}, 0.1 \%$ Triton, $0.2 \%$ NP-40, $6 \mathrm{mM}$ 2-mercapto-ethanol, proteinase inhibitor cocktail). The bound proteins were boiled and separated by sodium dodecyl sulfate-polyacrylamide gel electrophoresis. The presence of HA-RIN4 or HA-RIPK was detected by anti-HA.

\section{Luciferase complementation imaging (LCI) assays.}

LCI assays were performed as described previously (Chen et al. 2008). The coding sequences of $P B L s$ were inserted into pCAMBIA-nLUC and RIN4 or RIPK was inserted into pCAMBIAcLUC. All the constructs were transformed into Agrobacterium tumefaciens EHA105. An equal volume of A. tumefaciens harboring respective constructs were mixed and diluted to a final optical density at $600 \mathrm{~nm}$ of approximately 0.5 . After $48 \mathrm{~h}$ of A. tumefaciens expression in $N$. benthamiana, $0.5 \mathrm{mM}$ luciferin was sprayed on the leaves and a low-light cooled CCD imaging apparatus was used to capture the LUC image (iXon; Andor Technology).

\section{qRT-PCR analysis.}

Five-week-old plants were infiltrated with $P$. syringae pv. tomato DC3000 (EV) or DC3000 (avrB) at a concentration of $5 \times 10^{7} \mathrm{CFU} / \mathrm{ml}$. Total RNA was extracted by Trizol method (Invitrogen). First-strand cDNA was synthesized with $2 \mu \mathrm{g}$ of total RNA in a $25-\mu$ l reaction (Promega). qRT-PCR was performed according to manufacturer's manual (Toyobo). ACTIN2 was used as reference gene. All primers are listed in Supplementary Table S1.

\section{Kinase activity assays.}

PBL kinase activity assays were performed in $20 \mathrm{mM}$ Tris$\mathrm{HCl}$ (pH 7.5), $10 \mathrm{mM} \mathrm{MgCl} 2,1 \mathrm{mM} \mathrm{CaCl}_{2}, 10 \mu \mathrm{M}$ ATP, $1 \mu \mathrm{g}$ of kinase protein and 1 to approximately $2 \mu \mathrm{g}$ of recombinant HisRIN4 as substrates, in a total volume of $30 \mu$ l. Phosphorylated RIN4 was probed with anti-RIN4pT166 antibody (1:3,000). In planta RIN4 phosphorylation was performed as previously described (Liu et al. 2011).

\section{Bacterial growth assay.}

P. syringae pv. tomato DC3000 (EV), DC3000 (avrB), and DC3000 (avrRpm1) were grown on nutrient yeast glycerol agar plates at $28^{\circ} \mathrm{C}$ for $48 \mathrm{~h}$. Five-week-old plants were infiltrated with $P$. syringae pv. tomato DC3000 (EV), DC3000 (avrB), DC3000 (avrRpm1), or DC3000 (avrRps4) at $5 \times 10^{4} \mathrm{CFU} / \mathrm{ml}$. Bacterial populations were determined by growth curve analysis as described by Kim et al. (2005).

\section{Electrolyte leakage assay.}

To measure electrolyte leakage from $P$. syringae pv. tomato DC3000 (avrB)-infiltrated Arabidopsis leaves, eight leaf discs (6 $\mathrm{mm}$ in diameter) were collected from eight individual leaves. After washing three times with distilled water, the leaf discs were floated in $10 \mathrm{ml}$ of distilled water. Conductivity was monitored at the indicated time points.

\section{ACKNOWLEDGMENTS}

We thank G. Coaker (University of California, Davis) and D. Lu (Chinese Academy of Sciences) for their advices and critical reading of the manuscript. The study was supported by the Chinese Academy of Sciences (Strategic Priority Research program grant number XDB11020300) and the National Natural Science Foundation of China (grants 31570252 and 31500220). 


\section{LITERATURE CITED}

Afzal, A. J., da Cunha, L., and Mackey, D. 2011. Separable fragments and membrane tethering of Arabidopsis RIN4 regulate its suppression of PAMP-triggered immunity. Plant Cell 23:3798-3811.

Afzal, A. J., Kim, J. H., and Mackey, D. 2013. The role of NOI-domain containing proteins in plant immune signaling. BMC Genomics 14:327.

Baxter, L., Tripathy, S., Ishaque, N., Boot, N., Cabral, A., Kemen, E., Thines, M., Ah-Fong, A., Anderson, R., Badejoko, W., Bittner-Eddy, P., Boore, J. L., Chibucos, M. C., Coates, M., Dehal, P., Delehaunty, K., Dong, S., Downton, P., Dumas, B., Fabro, G., Fronick, C., Fuerstenberg, S. I., Fulton, L., Gaulin, E., Govers, F., Hughes, L., Humphray, S., Jiang, R. H., Judelson, H., Kamoun, S., Kyung, K., Meijer, H., Minx, P., Morris, P., Nelson, J., Phuntumart, V., Qutob, D., Rehmany, A., Rougon-Cardoso, A., Ryden, P., Torto-Alalibo, T., Studholme, D., Wang, Y., Win, J., Wood, J., Clifton, S. W., Rogers, J., Van den Ackerveken, G., Jones, J. D., McDowell, J. M., Beynon, J., and Tyler, B. M. 2010. Signatures of adaptation to obligate biotrophy in the Hyaloperonospora arabidopsidis genome. Science 330:1549-1551.

Bonardi, V., Cherkis, K., Nishimura, M. T., and Dangl, J. L. 2012. A new eye on NLR proteins: Focused on clarity or diffused by complexity? Curr. Opin. Immunol. 24:41-50.

Chen, H., Zou, Y., Shang, Y., Lin, H., Wang, Y., Cai, R., Tang, X., and Zhou, J. M. 2008. Firefly luciferase complementation imaging assay for protein-protein interactions in plants. Plant Physiol. 146:368-376.

Chisholm, S. T., Coaker, G., Day, B., and Staskawicz, B. J. 2006. Hostmicrobe interactions: Shaping the evolution of the plant immune response. Cell 124:803-814.

Chung, E. H., da Cunha, L., Wu, A. J., Gao, Z., Cherkis, K., Afzal, A. J., Mackey, D., and Dangl, J. L. 2011. Specific threonine phosphorylation of a host target by two unrelated type III effectors activates a host innate immune receptor in plants. Cell Host Microbe 9:125-136.

Chung, E. H., El-Kasmi, F., He, Y., Loehr, A., and Dangl, J. L. 2014. A plant phosphoswitch platform repeatedly targeted by type III effector proteins regulates the output of both tiers of plant immune receptors. Cell Host Microbe 16:484-494.

Coaker, G., Falick, A., and Staskawicz, B. 2005. Activation of a phytopathogenic bacterial effector protein by a eukaryotic cyclophilin. Science 308:548-550

Cui, H., Tsuda, K., and Parker, J. E. 2015. Effector-triggered immunity: From pathogen perception to robust defense. Annu. Rev. Plant Biol. 66:487-511.

Dangl, J. L., and Jones, J. D. 2001. Plant pathogens and integrated defence responses to infection. Nature 411:826-833.

de Torres, M., Sanchez, P., Fernandez-Delmond, I., and Grant, M. 2003. Expression profiling of the host response to bacterial infection: The transition from basal to induced defence responses in RPM1-mediated resistance. Plant J. 33:665-676.

Duan, C. G., Wang, C. H., Fang, R. X., and Guo, H. S. 2008. Artificial microRNAs highly accessible to targets confer efficient virus resistance in plants. J. Virol. 82:11084-11095.

Flor, H. H. 1971. Current status of the gene-for-gene concept. Annu. Rev. Phytopathol. 9:275-296.

Grant, M. R., Godiard, L., Straube, E., Ashfield, T., Lewald, J., Sattler, A., Innes, R. W., and Dangl, J. L. 1995. Structure of the Arabidopsis RPM1 gene enabling dual specificity disease resistance. Science 269:843-846.

Holt, B. F., 3rd, Belkhadir, Y., and Dangl, J. L. 2005. Antagonistic control of disease resistance protein stability in the plant immune system. Science 309:929-932.

Jones, J. D., and Dangl, J. L. 2006. The plant immune system. Nature 444:323-329.

Khan, M., Subramaniam, R., and Desveaux, D. 2016. Of guards, decoys, baits and traps: Pathogen perception in plants by type III effector sensors. Curr. Opin. Microbiol. 29:49-55.

Kim, M. G., da Cunha, L., McFall, A. J., Belkhadir, Y., DebRoy, S., Dangl, J. L., and Mackey, D. 2005. Two Pseudomonas syringae type III effectors inhibit RIN4-regulated basal defense in Arabidopsis. Cell 121:749-759.

Lee, D., Bourdais, G., Yu, G., Robatzek, S., and Coaker, G. 2015. Phosphorylation of the plant immune regulator RPM1-INTERACTING PROTEIN4 enhances plant plasma membrane $\mathrm{H}^{+}$-ATPase activity and inhibits flagellin-triggered immune responses in Arabidopsis. Plant Cell 27:2042-2056.
Li, M., Ma, X., Chiang, Y. H., Yadeta, K. A., Ding, P., Dong, L., Zhao, Y., Li, X., Yu, Y., Zhang, L., Shen, Q. H., Xia, B., Coaker, G., Liu, D., and Zhou, J. M. 2014. Proline isomerization of the immune receptorinteracting protein RIN4 by a cyclophilin inhibits effector-triggered immunity in Arabidopsis. Cell Host Microbe 16:473-483.

Lin, W., Lu, D., Gao, X., Jiang, S., Ma, X., Wang, Z., Mengiste, T., He, P., and Shan, L. 2013. Inverse modulation of plant immune and brassinosteroid signaling pathways by the receptor-like cytoplasmic kinase BIK1. Proc. Natl. Acad. Sci. U.S.A. 110:12114-12119.

Lin, Z. J., Liebrand, T. W., Yadeta, K. A., and Coaker, G. 2015. PBL13 is a serine/threonine protein kinase that negatively regulates Arabidopsis immune responses. Plant Physiol. 169:2950-2962.

Liu, J., Elmore, J. M., Fuglsang, A. T., Palmgren, M. G., Staskawicz, B. J., and Coaker, G. 2009. RIN4 functions with plasma membrane $\mathrm{H}^{+}$-ATPases to regulate stomatal apertures during pathogen attack. PLoS Biol. 7: e1000139.

Liu, J., Elmore, J. M., Lin, Z. J., and Coaker, G. 2011. A receptor-like cytoplasmic kinase phosphorylates the host target RIN4, leading to the activation of a plant innate immune receptor. Cell Host Microbe 9: 137-146.

Liu, Z., Wu, Y., Yang, F., Zhang, Y., Chen, S., Xie, Q., Tian, X., and Zhou, J. M. 2013. BIK1 interacts with PEPRs to mediate ethylene-induced immunity. Proc. Natl. Acad. Sci. U.S.A. 110:6205-6210.

Macho, A. P., and Zipfel, C. 2015. Targeting of plant pattern recognition receptor-triggered immunity by bacterial type-III secretion system effectors. Curr. Opin. Microbiol. 23:14-22.

Shang, Y., Li, X., Cui, H., He, P., Thilmony, R., Chintamanani, S., ZwieslerVollick, J., Gopalan, S., Tang, X., and Zhou, J. M. 2006. RAR1, a central player in plant immunity, is targeted by Pseudomonas syringae effector AvrB. Proc. Natl. Acad. Sci. U.S.A. 103:19200-19205.

Shao, F., Golstein, C., Ade, J., Stoutemyer, M., Dixon, J. E., and Innes, R. W. 2003. Cleavage of Arabidopsis PBS1 by a bacterial type III effector. Science 301:1230-1233.

Sun, X., Greenwood, D. R., Templeton, M. D., Libich, D. S., McGhie, T. K., Xue, B., Yoon, M., Cui, W., Kirk, C. A., Jones, W. T., Uversky, V. N., and Rikkerink, E. H. 2014. The intrinsically disordered structural platform of the plant defence hub protein RPM1-interacting protein 4 provides insights into its mode of action in the host-pathogen interface and evolution of the nitrate-induced domain protein family. FEBS J. 281: 3955-3979.

Tornero, P., Merritt, P., Sadanandom, A., Shirasu, K., Innes, R. W., and Dangl, J. L. 2002. RAR1 and NDR1 contribute quantitatively to disease resistance in Arabidopsis, and their relative contributions are dependent on the R gene assayed. Plant Cell 14:1005-1015.

Veronese, P., Nakagami, H., Bluhm, B., Abuqamar, S., Chen, X., Salmeron, J., Dietrich, R. A., Hirt, H., and Mengiste, T. 2006. The membrane-anchored BOTRYTIS-INDUCED KINASE1 plays distinct roles in Arabidopsis resistance to necrotrophic and biotrophic pathogens. Plant Cell 18: 257-273.

Wang, Y., Li, J., Hou, S., Wang, X., Li, Y., Ren, D., Chen, S., Tang, X., and Zhou, J. M. 2010. A Pseudomonas syringae ADP-ribosyltransferase inhibits Arabidopsis mitogen-activated protein kinase kinases. Plant Cell 22:2033-2044.

Zhang, J., Li, W., Xiang, T., Liu, Z., Laluk, K., Ding, X., Zou, Y., Gao, M., Zhang, X., Chen, S., Mengiste, T., Zhang, Y., and Zhou, J. M. 2010. Receptor-like cytoplasmic kinases integrate signaling from multiple plant immune receptors and are targeted by a Pseudomonas syringae effector. Cell Host Microbe 7:290-301.

Zhou, F., Mosher, S., Tian, M., Sassi, G., Parker, J., and Klessig, D. F. 2008. The Arabidopsis gain-of-function mutant ssi4 requires RARI and $S G T 1 b$ differentially for defense activation and morphological alterations. Mol Plant-Microbe Interact. 21:40-49.

Zipfel, C. 2014. Plant pattern-recognition receptors. Trends Immunol. 35: 345-351.

\section{AUTHOR-RECOMMENDED INTERNET RESOURCE}

The Arabidopsis Biological Resource Center: www.Arabidopsis.org 